\title{
Relationship between muscle strength and functional walking capacity among people with stroke
}

\author{
Carolina Moriello, MSc; ${ }^{*}$ Lois Finch, PhD; Nancy E. Mayo, PhD \\ Division of Clinical Epidemiology, Royal Victoria Hospital, Montreal, Canada; School of Physical and Occupational \\ Therapy, McGill University, Montreal, Canada
}

\begin{abstract}
The purpose of this study was to estimate, using the Position-Specific Global Index (PSGI) of Lower-Limb Muscle Strength, the extent to which muscle strength is independently associated with functional walking capacity. We performed an observational, cross-sectional study with 63 patients poststroke (onset between 3 and 12 months) at a major teaching hospital in an urban Canadian city. We assessed functional walking capacity with the six-minute walk test (6MWT) and muscle strength with handheld dynamometry. We established the relationship between the lower-limb muscles and functional walking capacity with the PSGI. The PSGI explained $70 \%(p<0.001)$ of the variability in the 6MWT, whereas the index of alternate against-gravity muscle strength explained $39 \%$ and the hip flexors in a supine position explained 51\%.
\end{abstract}

Key words: balance, comorbidity, functional walking capacity, lower limb, muscle strength, poststroke, six-minute walk test, spasticity, stroke, walking.

\section{INTRODUCTION}

Muscle weakness is a prevalent impairment of stroke, leading to activity limitations and participation restrictions [1]. A commonly limited activity is the ability to walk, with approximately 80 percent of people with stroke deficient in their walking ability at stroke onset [2].

Numerous stroke outcome studies have quantified the limitations in walking experienced by people with stroke [2-7]. The most common measure used to quantify functional walking capacity has been the six-minute walk test (6MWT), a task that requires walking speed and balance and submaximal endurance. These are the necessary components for community-dwelling people with stroke to live independently [2]. In a study by Salbach et al., a community-dwelling sample of people with stroke walked, upward of 1 year poststroke, an average of $207 \mathrm{~m}$ [2], which translates to 31 percent of normative values. Eng et al. demonstrated a similar finding where people with stroke walked, on average, $267 \mathrm{~m}$ [8].

Muscle strength has an important influence on functional walking capacity, and understanding the relationship between these two variables is critical to planning effective interventions to improve walking. Only a few studies have examined the relationship of muscle strength to functional walking capacity as measured by the 6MWT. The majority only assessed, or usually only reported, the relationship between a single muscle group and functional walking capacity [8-10]. For instance, Pang et al. demonstrated that among 63 people with stroke, at an average of 5.5 years poststroke, the strength

\footnotetext{
Abbreviations: $6 \mathrm{MWT}=$ six-minute walk test, $\mathrm{CSI}=\mathrm{Com}-$ posite Spasticity Index, MAS = Modified Ashworth Scale, PSGI = Position-Specific Global Index.

*Address all correspondence to Carolina Moriello, MSc; Division of Clinical Epidemiology, Ross Pavilion R4.27, Royal Victoria Hospital Site, 687 Pine Ave W, Montreal, QC Canada H3A 1A1; 514-943-1934, ext 36906; fax: 514-843-1493. Email: carolina.moriello@mcgill.ca

DOI:10.1682/JRRD.2010.04.0066
} 
of ankle plantar flexor muscles was a significant predictor of distance walked [10]. The information on the effect of single muscles is suspect because it is not always apparent from the research whether that muscle was the only one studied or if it emerged after an analysis involving multiple comparisons, in which case the finding may be considered a false positive. Basing a strengthening regimen on these findings would lead to a lack of efficacy. Moriello and Mayo presented a solution to this problem by developing a global index of lower-limb muscle strength derived from a principal component weighting of single muscles [11]. To identify which muscles are the most important for functional walking capacity, it would be impossible to incorporate the results of all the muscle strength tests in a single statistical analysis. This is where the Position-Specific Global Index (PSGI) of Lower-Limb Muscle Strength would be useful [11]. Furthermore, a possible useful adjunct would lead to implementing targeted rehabilitation interventions. Despite the important role muscle strength plays in functional walking capacity, it is not the only common consequence of stroke; decreased balance and spasticity are also influential [12-13].

As a person's muscle strength prior to stroke is unknown, poststroke muscle weakness can be ascertained only by comparing the strength of the affected side with the less affected side. Research indicates that the postulated differential between the muscles of the lower limbs reaches 40 to 50 percent [14-16].

The purpose of this study was twofold:

1. Estimate the extent to which lower-limb muscle strength is associated with functional walking capacity, taking into consideration three confounding variables: comorbidity, balance, and spasticity.

2. Identify which specific muscle groups in which position contributed the most to the relationship between strength and functional walking capacity, predicated on a significant relationship with the PSGI.

\section{METHODS}

\section{Participants}

The participants in this study were at least 3 months and no more than 1 year poststroke and recruited from a pool of people participating in two ongoing research studies. Detailed methods have been presented elsewhere [11]. Briefly, for this substudy participants were eligible if they were able to walk independently for $10 \mathrm{~m}$ with or without an aid and were discharged from active rehabilitation. Participants were excluded if they had any other neuromuscular condition or failed a cardiology stress test.

We based the sample size for this study on sufficient statistical power to detect a correlation coefficient of at least 0.50 as the lower limit of a desired 95 percent confidence interval. We estimated sample size according to the Fischer method based on correlation with a correction for additional variables $(n=\rho+k+1$, where $n=$ total number of participants, $\rho=$ calculated sample size based on desired correlation coefficient, and $k=$ number of additional variables measured) [17]. We required 60 participants to estimate a $\rho$ of 0.67 with a degree of precision that excludes values lower than 0.50 [18]. Given that two other variables, spasticity and balance $(k=2)$, were to be included as adjustment factors, we planned a sample size of 63 participants.

\section{Procedure}

We recorded age, sex, height, weight, side of hemiparesis, type of stroke, and time since onset of stroke for each participant. The primary outcome (dependent) variable was functional walking capacity, evaluated using the 6MWT. The variable under study (exposure or independent variable) was lower-limb muscle strength, and the potential confounding variables were balance, spasticity, and comorbidity.

\section{Functional Walking Capacity}

The 6MWT evaluates the capacity to maintain a moderate level of activity over a time period, reflective of activities of daily living [19-20] that are performed at a submaximal level of exertion [8]. We asked participants to walk as fast as they could for 6 minutes to cover as much distance as possible along a $15 \mathrm{~m}$ corridor. If needed, the participants were allowed to rest but had to continue walking as soon as possible. Each participant performed two trials, and we used the average of the two distances for the analysis.

\section{Muscle Strength}

The variable under study using the PSGI was lowerlimb muscle strength. The muscle groups we assessed were the hip flexors and hip extensors, and the separate muscles we tested were the knee flexors, knee extensors, ankle dorsiflexor, and ankle plantar flexors (gastrocnemius 
and soleus). We assessed the muscles by using standardized methodology in different positions (against-gravity, alternate against-gravity, and gravity-eliminated) on the affected and less affected sides, a total of 96 musclestrength data points. From these points we summarized the lower-limb muscle strength into five indexes (two for the affected side and three for the unaffected side) with two subindexes. We summarized the muscle strength of the affected side in index 1(a): against-gravity position, index 1(b): alternate against-gravity position, and index 2: gravity-eliminated position. We summarized the muscle strength of the less affected side in index 3(a): againstgravity position, 3(b): alternate against-gravity position, 4: gravity-eliminated hip and knee position, and 5: gravityeliminated ankle position. To provide a single value for lower-limb muscle strength, we further reduced all the indexes, representing both the affected and less affected sides of the body, to the PSGI. Full details regarding the index can be found in the original article [11].

\section{Balance}

We assessed balance by using the Berg Balance Scale. The scale consists of 14 items (maximum of 56 points), using a 5-point scale (0-4) to rate each item. A score of 0 indicates the need for maximal assistance to perform the tasks on the scale and a score of 4 indicates independence and the ability to safely perform the tasks. The tasks require the participant to maintain positions with a variety of difficulty, such as transferring and balancing [21].

\section{Spasticity}

We measured spasticity using the Modified Ashworth Scale (MAS) and the Composite Spasticity Index (CSI). The MAS uses a 6-point scale to assess the degree of resistance encountered during passive muscle stretching [22]. The score is further used to classify levels of spasticity: none, mild, moderate, and severe. The CSI is a clinical measure of spasticity that also classifies spasticity as none, mild, moderate, and severe [23]. For the purposes of this analysis, we created a dichotomous variable for each section: present or absent. We considered spasticity present if we classified individuals in the mild, moderate, or severe spasticity categories on both measures. We considered spasticity absent if we classified individuals as having no spasticity on both measures.

\section{Comorbidity}

We obtained information on comorbidity from the participant's medical chart or primary care physician. We used the total number from a list of conditions known to be prevalent among people with stroke in the analysis.

\section{Data Analysis}

Basic statistics helped describe the characteristics of the study participants and the distributions of the variables. The first step was to assess the effect of balance, spasticity, and comorbidity on the outcome, the value of the 6MWT averaged over two trials, and lower-limb muscle strength as assessed by the PSGI. We used simple linear regression for these analyses and retained only the variables significantly associated with both the outcome and the variable under study. We subsequently used multiple linear regression to estimate the association between the PSGI and functional walking capacity, adjusting for the significant confounders.

If the above association between the outcome and the variables under study was statistically significant, the second step was to estimate the association between the $6 \mathrm{MWT}$ and each of the muscle-strength indexes. The third step estimated the relationship between 6MWT and the strength of the individual muscles within each of the indexes for those associations between the 6MWT and an index that were significant. For the latter two analyses (steps 2 and 3), we used stepwise linear regression with the confounders forced into the model.

\section{RESULTS}

We assessed the muscle strength of a total of 63 people with chronic stroke. Table 1 provides the demographic characteristics of the participant sample. The average age of participants was 67 years and 68 percent were male; on average, participants walked $277 \mathrm{~m}$ on the 6MWT, approximately 42 percent of their predicted distance [24].

Table 2 provides the muscle strength for each muscle or muscle group and position. In contrast with the less affected side, the affected side was 8 to 21 percent weaker, which was even more evident when tested in against-gravity positions. Compared with normative data on muscle strength adjusted for age and sex, the weakness differential increased to 22 to 88 percent depending on muscle and position [25-27].

Three confounding variables (comorbidity, balance, and spasticity) were statistically significant in simple 
JRRD, Volume 48, Number 3, 2011

regression analyses with the variable under study (PSGI)

Table 1.

Participant demographic characteristics $(n=63)$.

\begin{tabular}{|c|c|c|c|c|c|}
\hline Characteristic & Mean \pm SD & Median & Range & $\mathrm{CV}$ & $\boldsymbol{n}$ \\
\hline$\overline{\text { Age (yr) }}$ & $67.0 \pm 12.3$ & 68.0 & $36.0-93.0$ & 18.3 & - \\
\hline \multicolumn{6}{|l|}{ Sex } \\
\hline Male & - & - & - & - & 43 \\
\hline Female & - & - & - & - & 20 \\
\hline \multicolumn{6}{|l|}{ Type of Stroke } \\
\hline Ischemic & - & - & - & - & 53 \\
\hline Hemorrhagic & - & - & - & - & 10 \\
\hline Onset Since Stroke (mo) & $3.9 \pm 1.6$ & - & - & - & - \\
\hline \multicolumn{6}{|l|}{ Affected Side } \\
\hline Right & - & - & - & - & 25 \\
\hline Left & - & - & - & - & 32 \\
\hline None & - & - & - & - & 6 \\
\hline 6MWT (m) & $277 \pm 136$ & 293 & $0-623$ & 49.0 & - \\
\hline Berg Balance Test (score) ${ }^{*}$ & $48.0 \pm 10.5$ & 52.0 & $7.0-56.0$ & 21.8 & - \\
\hline \multicolumn{6}{|l|}{ Spasticity } \\
\hline Yes & - & - & - & - & 26 \\
\hline No & - & - & - & - & 37 \\
\hline Gait Speed (m/s) & $0.89 \pm 0.38$ & 0.91 & $0.00-1.82$ & 42.80 & - \\
\hline
\end{tabular}

and outcome (6MWT). We used these variables in the subsequent multiple regression analyses.

The PSGI (against-gravity position) was a significant correlate of the 6MWT distance. The simple regression demonstrated that the PSGI alone explained 29 percent $(p<$ 0.001 ) of the variability in the 6MWT. The PSGI, including the alternate against-gravity position, explained 28 percent $(p<0.001)$ of the variability. There was a negligible difference between both PSGI scores.

When we added the confounders (comorbidity, balance, and spasticity) to the model, 70 percent $(p<0.001)$ of the variability in the 6MWT was explained. In the full model, the PSGI remained a significant predictor of the 6MWT (Table 3) but spasticity and comorbidity were no longer significant predictors. Balance remained an independent associate of the 6MWT.

As the PSGI was a significant contributing factor, the next step was to assess the relationship of each of the indexes within the PSGI to functional walking capacity. All five indexes correlated significantly with the distance walked (Table 4). The against-gravity position on the affected side (index 1(a)) explained 38 percent of the variability $\left(r^{2}=0.38, p<0.001\right)$. The hip flexors within this index explained more of the variability (44\%) than other muscles (Table 5). The alternate against-gravity position index (index 1(b)) explained the most variability $\left(r^{2}=0.39, p<0.001\right)$ of the functional walking capacity when compared with the other indexes. Again, the hip flexors within this index explained 51 percent $(p<0.001)$ of variability more than the other muscles in the index.

The affected gravity-eliminated position index (index 2) was a significant correlate of the 6MWT $\left(r^{2}=0.24, p<\right.$ 0.001 ), with the ankle plantar flexor (gastrocnemius) explaining the most variability (23\%).

The less affected against-gravity position index (index 3(a)) explained 20 percent of the variability of walking $(p<0.001)$. Again, the hip flexors in the againstgravity position for the less affected side were a significant correlate of functional walking capacity compared with the other muscles within the index. The alternate against-gravity position index (index 3(b)) explained slightly less variability $\left(r^{2}=0.18, p=0.01\right)$, and the hip extensors were a significant correlate of functional walking capacity. 
Table 2.

Muscle strength (kilograms) for each position on affected and less affected sides.

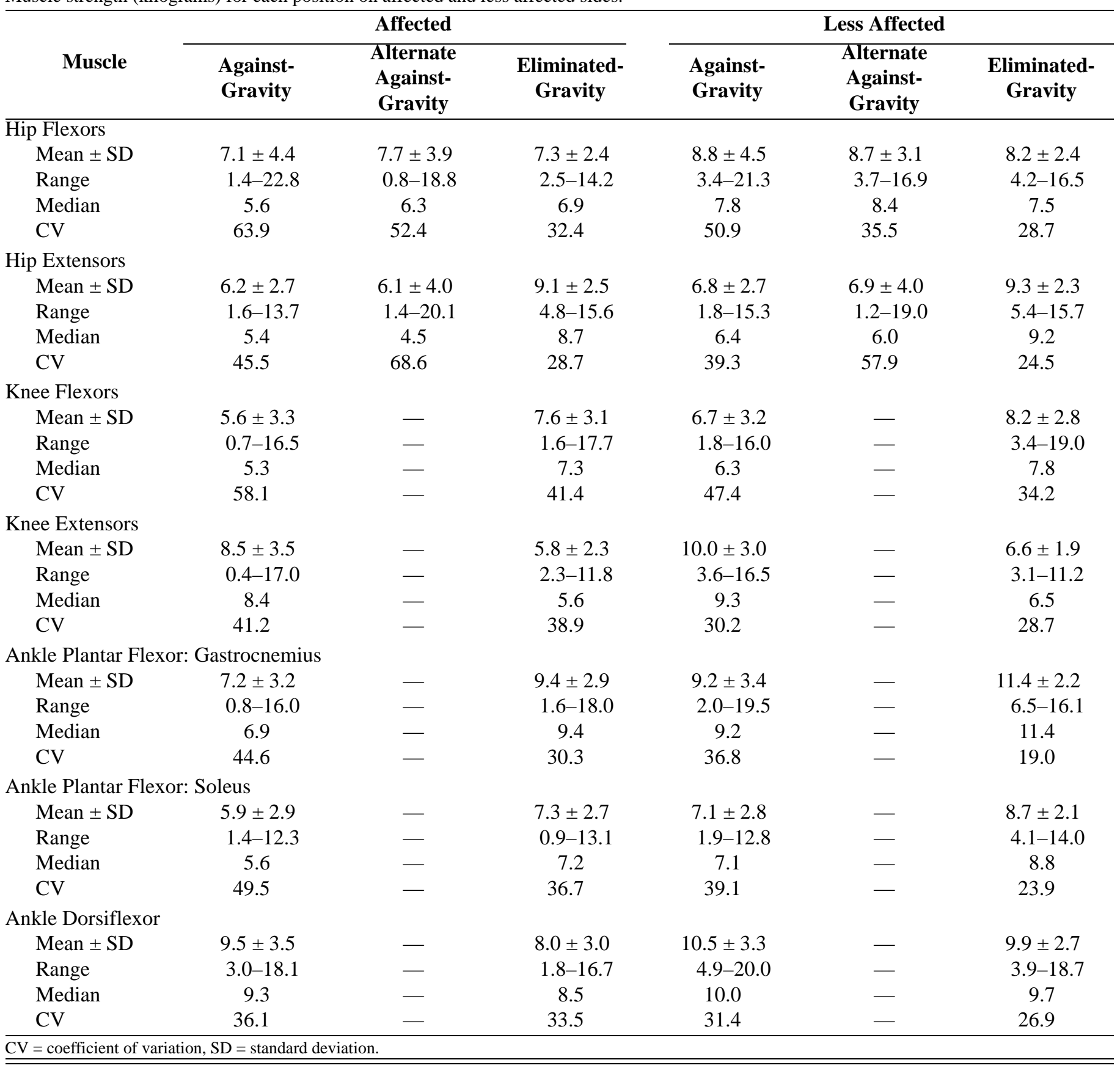

The least variability explained was by the less affected gravity-eliminated position index with muscle strength of the hip and knee (index 4, $r^{2}=0.09, p=0.02$ ). The knee flexors and knee extensors of this index explained 20 percent of the variability of walking.

The less affected gravity-eliminated position index with muscles of the ankle (index 5) was a significant correlate of the 6MWT $\left(r^{2}=0.24, p<0.001\right)$, with the ankle plantar flexors (gastrocnemius) explaining 17 percent of variability.

The significant relationship of the PSGI with the position-specific indexes allows for testing of the relationship between the individual muscles and the 6MWT (Table 5). The greatest variability in the 6MWT was explained by the hip flexors in an alternate against-gravity 
JRRD, Volume 48, Number 3, 2011

Table 3.

Multiple regression of Position-Specific Global Index (PSGI) of Lower-Limb Muscle Strength comorbidity, balance, and spasticity to six-minute walk test.

\begin{tabular}{lcr}
\hline \multicolumn{1}{c}{ Variable } & $\boldsymbol{\beta} \pm \mathbf{S E}$ & $\boldsymbol{p}$-Value \\
\hline PSGI & $0.56 \pm 0.25$ & 0.03 \\
No. of Comorbid Conditions & $-36.30 \pm 19.60$ & 0.07 \\
Balance & $8.20 \pm 1.05$ & $<0.001$ \\
Spasticity & $-33.60 \pm 20.50$ & 0.11 \\
${ }^{*} r^{2}=0.70, p<0.001$. & & \\
SE $=$ standard error. & & \\
\hline \hline
\end{tabular}

Table 4.

Relationship between Position-Specific Global Index (PSGI) of Lower-Limb Muscle Strength and six-minute walk test by regression analysis done to demonstrate the contribution of muscle strength to walking (meters).

\begin{tabular}{|c|c|c|c|}
\hline Index & $\beta \pm \mathrm{SE}$ & $r^{2}$ & $p$-Value \\
\hline PSGI & $1.70 \pm 0.32$ & 0.29 & $<0.001$ \\
\hline \multicolumn{4}{|l|}{ Affected Side } \\
\hline 1(a).Against-Gravity & $4.90 \pm 0.82$ & 0.38 & $<0.001$ \\
\hline 2. Gravity-Eliminated & $5.60 \pm 1.27$ & 0.24 & $<0.001$ \\
\hline \multicolumn{4}{|l|}{ Less Affected Side } \\
\hline 4. Gravity-Eliminated (Hip and Knee) & $6.80 \pm 2.70$ & 0.09 & 0.02 \\
\hline 5. Gravity-Eliminated (Ankle) & $16.60 \pm 3.80$ & 0.24 & $<0.001$ \\
\hline
\end{tabular}

Table 5.

Relationship between individual muscles by position (affected side) and six-minute walk test by regression analysis done to demonstrate the contribution of muscle strength to walking (meters).

\begin{tabular}{|c|c|c|c|c|c|c|c|c|c|}
\hline \multirow{2}{*}{ Muscle } & \multicolumn{3}{|c|}{ Against-Gravity } & \multicolumn{3}{|c|}{ Alternate Against-Gravity } & \multicolumn{3}{|c|}{ Gravity-Eliminated } \\
\hline & $\boldsymbol{B} \pm \mathrm{SE}$ & $r^{2}$ & $p$-Value & $\beta \pm S E$ & $r^{2}$ & $\overline{p \text {-Value }}$ & $\beta \pm S E$ & $r^{2}$ & $p$-Value \\
\hline Hip Flexors & $20.2 \pm 2.9$ & 0.44 & $<0.001$ & $24.5 \pm 3.1$ & 0.51 & $<0.001$ & $20.7 \pm 6.9$ & 0.13 & 0.01 \\
\hline Hip Extensors & $23.1 \pm 5.6$ & 0.22 & $<0.001$ & $16.3 \pm 3.8$ & 0.23 & $<0.001$ & $21.5 \pm 6.2$ & 0.16 & 0.01 \\
\hline Knee Flexors & $21.0 \pm 4.6$ & 0.25 & $<0.001$ & - & - & - & $15.8 \pm 5.2$ & 0.13 & 0.01 \\
\hline Knee Extensors & $16.5 \pm 4.5$ & 0.18 & 0.01 & - & - & - & $18.9 \pm 7.4$ & 0.09 & 0.01 \\
\hline Ankle Dorsiflexor & $20.9 \pm 4.3$ & 0.28 & $<0.001$ & - & - & - & $15.8 \pm 5.4$ & 0.12 & 0.01 \\
\hline Soleus & $20.7 \pm 5.5$ & 0.19 & $<0.001$ & - & - & - & $22.9 \pm 5.9$ & 0.19 & $<0.001$ \\
\hline
\end{tabular}

position $\left(r^{2}=0.51 ; p<0.001\right)$, followed by the hip flexors in an against-gravity position $\left(r^{2}=0.44 ; p<0.001\right)$.

The next step in the analyses looked at the individual muscles using a stepwise regression. The results demonstrated that hip flexor strength in the alternate againstgravity position was the only muscle significantly associated with the distance walked $(\beta=24.5$; standard error $=3.1)$. The hip flexors explained 51 percent of the variability in the 6MWT, controlling for levels of balance, comorbidity, and spasticity.

\section{DISCUSSION}

Stroke had a devastating effect on the muscle strength of both the affected and less affected lower limbs of the participants in this study. A large difference in the 
percentage of muscle weakness when comparing with the less affected side $(8 \%-22 \%)$ or with the standards of muscle strength (22\%-88\%) was demonstrated. This emphasizes that stroke has an effect on the less affected side.

The significance of the PSGI in the regression analyses justified assessing the relationship between the various measures of strength and functional walking capacity. Results indicated that muscle strength, no matter in what position tested, contributed to the variability in the $6 \mathrm{MWT}$. This supports not only task-specific training to increase the distance walked but also muscle strength training in different positions. Additionally, it supports that global strength measured by a single index incorporating all the lower-limb muscles was strongly associated with distance walked; it also reinforces the concept of specific strength-training programs for individual muscles to improve walking in addition to training on functional tasks that improve overall muscle strength. For example, a training program of walking tasks involving walking up and down stairs or inclines or while carrying loads is likely to yield improvements in functional walking capacity by increasing the global strength of the lower limb. Indeed, in a recent randomized, controlled trial, Salbach et al. found such a program to increase the distance walked on the 6MWT by 40 percent compared with 0 percent with an equal-attention control intervention of upper-limb tasks [2].

Each index had specific muscles that contributed more to explaining the variability in functional walking capacity on the more affected side. The strength of the hip flexors in the against- or alternate against-gravity positions explained the most variability; in the gravityeliminated position, gastrocnemius strength explained the most variability in functional walking capacity. This finding is not surprising because the literature has demonstrated the importance of the gastrocnemius [9,28-30], but it further supports the importance of this muscle in rehabilitation through functional training. Although this study further supports the findings from other studies, it also provides a novel approach to determining the relationship between muscle strength and functional walking capacity by including numerous muscles.

In regards to the muscles on the less affected side, the hip flexors and extensors explain the most variability in the against- and alternate against-gravity positions, respectively. For the gravity-eliminated positions, the knee flexors and extensors explain the most variability for the hip and knee and the ankle plantar flexors (gastrocnemius) explain the most variability for the ankle.

While controlling for the confounding variables, we found the hip flexors on an alternate against-gravity position (supine position) to be the strongest contributor to the variability of functional walking capacity. Supine most closely simulates the upright position where the hip flexors are needed to initiate the swing phase of gait from a hip-extended position. Increasing the strength of the hip flexors in the supine position would be an ideal intervention in rehabilitation to improve functional walking capacity.

\section{CONCLUSIONS}

Clinically, this study can help optimize both treatment of patients and time spent by therapists. During an assessment, a therapist now has the option of assessing only the gravity-related muscles (hip flexors, gastrocnemius, tibialis anterior) or at a minimum, the hip flexors in an alternate against-gravity position. Potential treatment approaches to improve functional walking capacity may include global strength training through walking tasks, strength training in different positions, and emphasis on strengthening hip flexors in the supine position.

The PSGI allowed us to include and assess multiple muscles. Walking necessitates the use of all lower-limb muscles to some degree. Only the PSGI can determine the relationship between all lower-limb muscles and walking among people with stroke. It also provides a numerical indication of the contribution of each muscle to walking. Without the PSGI, a statistical analysis that provided a better insight into the relationship between strength and walking would be compromised by a restricted set of variables.

\section{ACKNOWLEDGMENTS}

\section{Author Contributions:}

Study concept and design: N. E. Mayo, C. Moriello. Acquisition of data: C. Moriello, L. Finch.

Analysis and interpretation of data: N. E. Mayo, C. Moriello. Drafting of manuscript: N. E. Mayo, L. Finch, C. Moriello. Critical revision of manuscript for important intellectual content: N. E. Mayo, L. Finch.

Statistical analysis: C. Moriello.

Administrative, technical, or material support: L. Finch.

Study supervision: N. E. Mayo. 
Financial Disclosures: The authors have declared that no competing interests exist.

Funding/Support: This material was unfunded at the time of manuscript preparation.

Institutional Review: All studies received approval from the local ethics committee. Participants consented to the muscle testing within the studies.

Participant Follow-Up: The authors have notified the healthcare professionals from which the participants were recruited, but we will not be contacting the participants directly because of a lack of contact information.

\section{REFERENCES}

1. Ng S, Shepherd R. Weakness in patients with stroke: Implications for strength training in neurorehabilitation. Phys Ther Rev. 2000;5(4):227-38.

2. Salbach NM, Mayo NE, Wood-Dauphinee S, Hanley JA, Richards CL, Côté R. A task-orientated intervention enhances walking distance and speed in the first year poststroke: A randomized controlled trial. Clin Rehabil. 2004; 18(5):509-19. [PMID: 15293485] DOI:10.1191/0269215504cr763oa

3. Dean CM, Richards CL, Malouin F. Walking speed over 10 metres overestimates locomotor capacity after stroke. Clin Rehabil. 2001;15(4):415-21. [PMID: 11518442] DOI:10.1191/026921501678310216

4. Mayo NE, Wood-Dauphinee S, Ahmed S, Gordon C, Higgins J, McEwen S, Salbach N. Disablement following stroke. Disabil Rehabil. 1999;21(5-6):258-68. [PMID: 10381238]

5. Eng JJ, Dawson AS, Chu KS. Submaximal exercise in persons with stroke: Test-retest reliability and concurrent validity with maximal oxygen consumption. Arch Phys Med Rehabil. 2004;85(1):113-18. [PMID: 14970978]

DOI:10.1016/S0003-9993(03)00436-2

6. Duncan P, Richards L, Wallace D, Stoker-Yates J, Pohl P, Luchies C, Ogle A, Studenski S. A randomized, controlled pilot study of a home-based exercise program for individuals with mild and moderate stroke. Stroke. 1998;29(10):2055-60. [PMID: 9756581]

7. Duncan P, Studenski S, Richards L, Gollub S, Lai SM, Reker D, Perera S, Yates J, Koch V, Rigler S, Johnson D. Randomized clinical trial of therapeutic exercise in subacute stroke. Stroke. 2003;34(9):2173-80. [PMID: 12920254$]$ DOI:10.1161/01.STR.0000083699.95351.F2

8. Eng JJ, Chu KS, Dawson AS, Kim CM, Hepburn KE. Functional walk tests in individuals with stroke: Relation to perceived exertion and myocardial exertion. Stroke. 2002; 33(3):756-61. [PMID: 11872900]

DOI:10.1161/hs0302.104195

9. Pohl PS, Perera S, Duncan PW, Maletsky R, Whitman R, Studenski S. Gains in distance walking in a 3-month follow- up poststroke: What changes? Neurorehabil Neural Repair. 2004;18(1):30-36. [PMID: 15035962]

DOI:10.1177/0888439003260494

10. Pang MY, Eng JJ, Dawson AS. Relationship between ambulatory capacity and cardiorespiratory fitness in chronic stroke: Influence of stroke-specific impairments. Chest. 2005; 127(2):495-501. [PMID: 15705987]

DOI:10.1378/chest.127.2.495

11. Moriello C, Mayo NE. Development of a position-specific index of muscle strength to be used in stroke evaluation. Arch Phys Med Rehabil. 2006;87(11):1490-95.

[PMID: 17084125]

DOI:10.1016/j.apmr.2006.07.261

12. Niam S, Cheung W, Sullivan PE, Kent S, Gu X. Balance and physical impairments after stroke. Arch Phys Med Rehabil. 1999;80(10):1227-33. [PMID: 10527078] DOI:10.1016/S0003-9993(99)90020-5

13. Ada L, Vattanasilp W, O’Dwyer NJ, Crosbie J. Does spasticity contribute to walking dysfunction after stroke? J Neurol Neurosurg Psychiatry. 1998;64(5):628-35. [PMID: 9598679] DOI:10.1136/jnnp.64.5.628

14. Newham DJ, Hsiao SF. Knee muscle isometric strength, voluntary activation and antagonist co-contraction in the first six months after stroke. Disabil Rehabil. 2001;23(9): 379-86. [PMID: 11394588] DOI:10.1080/0963828001006656

15. Andrews AW, Bohannon RW. Distribution of muscle strength impairments following stroke. Clin Rehabil. 2000;14(1): 79-87. [PMID: 10688348] DOI:10.1191/026921500673950113

16. Hsu AL, Tang PF, Jan MH. Analysis of impairments influencing gait velocity and asymmetry of hemiplegic patients after mild to moderate stroke. Arch Phys Med Rehabil. 2003;84(8):1185-93. [PMID: 12917858] DOI:10.1016/S0003-9993(03)00030-3

17. Kraemer HC, Thiemann S. How many subjects?: Statistical power analysis in research. Newbury Park (CA): Sage Publications; 1989.

18. Lowry R. The confidence interval of rho [Internet]. Poughkeepsie (NY): Vassar College; 2010. Available from: http://faculty.vassar.edu/lowry/rho.html

19. Eng JJ, Chu KS. Reliability and comparison of weightbearing ability during standing tasks for individuals with chronic stroke. Arch Phys Med Rehabil. 2002;83(8):1138-44. [PMID: 12161837] DOI:10.1053/apmr.2002.33644

20. Finch E. Physical rehabilitation outcome measures: A guide to enhanced clinical decision making. 2nd ed. Hamilton (Canada): BC Decker; 2002.

21. Berg K, Wood-Dauphinee S, Williams JI. The Balance Scale: Reliability assessment with elderly residents and 
patients with an acute stroke. Scand J Rehabil Med. 1995; 27(1):27-36. [PMID: 7792547]

22. Blackburn M, Van Vliet P, Mockett SP. Reliability of measurements obtained with the Modified Ashworth Scale in the lower extremities of people with stroke. Phys Ther. 2002;82(1):25-34. [PMID: 11784275]

23. Jobin A, Levin MF. Regulation of stretch reflex threshold in elbow flexors in children with cerebral palsy: A new measure of spasticity. Dev Med Child Neurol. 2000;42(8): 531-40. [PMID: 10981931] DOI:10.1017/S0012162200001018

24. Gibbons WJ, Fruchter N, Sloan S, Levy RD. Reference values for a multiple repetition 6-minute walk test in healthy adults older than 20 years. J Cardiopulm Rehabil. 2001;21(2):87-93. [PMID: 11314289] DOI:10.1097/00008483-200103000-00005

25. Phillips BA, Lo SK, Mastaglia FL. Muscle force measured using "break" testing with a hand-held myometer in normal subjects aged 20 to 69 years. Arch Phys Med Rehabil. 2000; 81(5):653-61. [PMID: 10807107]

26. Andrews AW, Thomas MW, Bohannon RW. Normative values for isometric muscle force measurements obtained with hand-held dynamometers. Phys Ther. 1996;76(3): 248-59. [PMID: 8602410$]$

27. Bohannon RW. Reference values for extremity muscle strength obtained by hand-held dynamometry from adults aged 20 to 79 years. Arch Phys Med Rehabil. 1997;78(1): 26-32. [PMID: 9014953] DOI:10.1016/S0003-9993(97)90005-8
28. Hsu AL, Tang PF, Jan MH. Test-retest reliability of isokinetic muscle strength of the lower extremities in patients with stroke. Arch Phys Med Rehabil. 2002;83(8):1130-37. [PMID: 12161836$]$

DOI:10.1053/apmr.2002.33652

29. Nadeau S, Arsenault AB, Gravel D, Bourbonnais D. Analysis of the clinical factors determining natural and maximal speeds in adults with stroke. Am J Phys Med Rehabil. 1999; 78(2):123-30. [PMID: 10088586] DOI:10.1097/00002060-199903000-00007

30. Nadeau S, Gravel D, Arsenault AB, Bourbonnais D. Plantarflexor weakness as a limiting factor of gait speed in stroke subjects and the compensating role of hip flexors. Clin Biomech (Bristol, Avon). 1999;14(2):125-35.

[PMID: 10619100] DOI:10.1016/S0268-0033(98)00062-X

Submitted for publication April 14, 2010. Accepted in revised form November 10, 2010.

This article and any supplementary material should be cited as follows:

Moriello C, Finch L, Mayo NE. Relationship between muscle strength and functional walking capacity among people with stroke. J Rehabil Res Dev. 2011;48(3):267-76. DOI:10.1682/JRRD.2010.04.0066

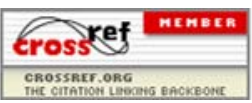


